\title{
Generic behaviours in impact fragmentation
}

\author{
N. Sator ${ }^{1}$, S. Mechkov ${ }^{\star \ddagger}$ and F. Sausset* \\ * Laboratoire de Physique Théorique de la Matière Condensée, Université \\ Pierre et Marie Curie (Paris 6), UMR CNRS 7600 - 4 place Jussieu, 75252 \\ Paris Cedex 05, France, \\ ¥ Laboratoire de Physique Statistique, Ecole Normale Supérieure - 24 rue \\ Lhomond, 75231 Paris Cedex 05, France
}

October 31, 2018

\begin{abstract}
We present a simple numerical model for investigating the general properties of fragmentation. By use of molecular dynamics simulations, we study the impact fragmentation of a solid disk of interacting particles with a wall. Regardless of the particular form of the interaction potential, the fragment size distribution exhibits a power law behaviour with an exponent that increases logarithmically with the energy deposited in the system, in agreement with experiments. We expect this behaviour to be generic in fragmentation phenomena.
\end{abstract}

PACS numbers: 46.50.+a, 62.20.Mk, 89.75.Da

Keywords: fragmentation, cracks, scaling laws

\section{Introduction}

From atomic nuclei to supernovae, including plates and rocks, every cohesive system can be broken into fragments, provided that the deposited energy is sufficiently large compared to its cohesive energy [1]. The ubiquity and the fundamental role of fragmentation are reflected in planetary formation [2] and geological phenomena 3 like explosive volcanic eruptions [4, as well as in the determination of the phase diagram of nuclear matter [5, 6]. Furthermore, a major part of activities in building and pharmaceutical industries makes use of particle size reduction processes [1, 7.

In spite of this wide variety of length scales and materials, fragmentation processes present some generic features. The most striking of them is the frequently observed power law form of the fragment size distribution, whose exponent $\tau$ varies from 1 to about 2.5 according to the data provided by experiments [1, 3, 5, 6, 9, 10, 11, 17, 23, 24, 25, 30, 32, 33, 34, 36 and natural phenomena [3, 4, 8].

Over the last decades, much effort has been devoted to understanding the origin of this scaling behaviour and a number of proposals have been advanced. The scattering of the values of $\tau$ casts some doubts [9, 10] on a self-organized critical mechanism proposed by Oddershede et al. [11, but raises the question of the existence of universality classes in fragmentation [12, 13. Thus, collision experiments of atomic nuclei exhibit a percolation behaviour of the fragment

\footnotetext{
${ }^{1}$ sator@lptmc.jussieu.fr
} 
size distribution $(\tau \simeq 2.2)[6$, which is typical of the fragmentation of supercritical fluids 14. Likewise, evidence for criticality has been found in hydrogen cluster fragmentation [15. In contrast, the definition of universality classes in fragmentation of macroscopic systems is still an open question.

As matter stands, fragmentation may depend on several parameters like the dimension of the space, the shape, size and material of the fragmenting object, as well as on the way it is broken and on the amount of energy deposited in it. Simple analytical models based on rate equations [16, binary fissions 9 , or sequential fragmentation [17, 18] although elegant and instructive have limitations and cannot take into account all the above-mentioned parameters. As an example, most of the analytical models predict a single exponent $\tau$ depending only on the dimension of the space, which experiments seem to refute.

Molecular dynamics calculations have been proven to be a powerful technique for addressing the role played by the parameters that may influence fragmentation processes. Furthermore, simulations allow one to study the dynamics of an irreversible and strongly out-of-equilibrium process like fragmentation. Until now, mainly two kinds of numerical experiments have been proposed. On the one hand, explosive fragmentation of a system is simulated by initially allocating a centrifugal velocity to the particles, which interact through a Lennard-Jones potential [10, 13, 19, 20]. On the other hand, various numerical simulations are concerned with the impact fragmentation of brittle solids [12, 13, 26, 28, 29. Of particular interest, Kun and Herrmann proposed a sophisticated model that takes into account elastic, shear and torque interactions between particles of a two-dimensional disk which impacts another disk 12 or a hard plate 28 . They suggested that fragmentation of solids occurs as a continuous phase transition from a damaged state to a fragmented state when the energy deposited in the system increases.

In the same spirit, we propose a simple model providing a general framework for investigating the role of the different parameters that may be relevant in fragmentation processes. In this paper, we study the impact fragmentation of a two-dimensional disc of interacting particles with a wall, as a function of the energy deposited in the system. The role played by the materials (interaction potential) and by the size of the disc (number of particles) are especially investigated.

Note that although fragmenting systems are usually three-dimensional, some experiments are performed on platelike objects with a two-dimensional experimental setup [17, 30. Furthermore, we are here concerned with general problems that may be tackled by two-dimensional simulations.

\section{Model}

To provide a generic frame of reference, the fragmenting system is made up of particles which represent mesoscopic grains of materials, without specifying their exact nature. These particles interact through a two-body central LennardJones type potential:

$$
\mathrm{v}\left(\mathrm{r}_{\mathrm{ij}}\right)=v_{0} \epsilon\left[\left(\frac{\sigma}{\mathrm{r}_{\mathrm{ij}}}\right)^{a}-\left(\frac{\sigma}{\mathrm{r}_{\mathrm{ij}}}\right)^{b}\right],
$$


where $\mathrm{r}_{\mathrm{ij}}$ is the distance between particles $i$ and $j$, and the two constants, $\epsilon$ and $\sigma$, set the energy and length scales respectively. Hence, $\sigma$ is typically the diameter of the particles. The two exponent parameters, $a$ and $b$, control the range of attraction and $v_{0}$ is used to set the minimum of the potential to $-\epsilon$. In order to mimic the cohesive interaction at a mesoscopic scale, we choose a very short-range potential $\left(v_{0}=107.37, a=80\right.$ and $\left.b=78\right)$ with a typical range of attraction as short as $0.1 \sigma$, in addition to the particle diameter. As we will see in the following, our results are not sensitive to the particular choice of the parameters $v_{0}, a$ and $b$, that is to the range of attraction.

At first, a disc of a given number of particles is cut in a two-dimensional triangular lattice with a lattice spacing corresponding to the minimum of the interaction potential given by eq. (1). The fragmenting disc is thus a perfect crystal (without any quenched disorder) prepared at zero temperature and at the corresponding number density of $\rho \sigma^{2} \simeq 1.16$. Next, the disc is rotated by a random angle and launched towards a wall, as shown in fig. 1 1 , by assigning to each particle a given impact velocity $\mathbf{V}$, perpendicular to the wall. We use the magnitude of the impact velocity as a natural control parameter. The particles of the disc interact with the wall through the repulsive part of the potential given by eq. (1), that is $v_{0} \epsilon\left(\sigma / z_{i}\right)^{a}$, where $z_{i}$ is the distance between the wall and the particle $i$. We checked that our results do not depend on the precise form of this hard-core potential.

To study the fragmentation process and its outcomes, we perform molecular dynamics simulations at constant energy using the Verlet algorithm 21. The time step is $\delta t=0.0005 t_{0}$, where $t_{0}=\sqrt{\epsilon \sigma^{2} / m}$ is the unit of time and $m$ the particle mass, which ensures the conservation of the total energy within $0.01 \%$ of its average value. In the following, times and velocities are expressed in units of $t_{0}$ and $\sigma / t_{0}$ respectively. As we will see below, at $t=500$ (10 ${ }^{6}$ iterations), the fragmentation process has already reached a steady state. The main results presented in this paper are obtained with a disc of $N=1457$ particles, but system sizes up to $N=36289$ were used with a view to determine the finite size scaling properties of the system. For $N=1457$, the potential energy per particle is $-2.89 \epsilon$, slightly in excess of the bulk value $(-3 \epsilon)$, because of surface effects.

During the fragmentation process, fragments are identified as being selfbound clusters of particles 14, 22. More precisely, two particles are linked if their relative kinetic energy is lower than the absolute value of their interaction energy. Note that a definition of clusters based on an arbitrary cutoff distance provides the same results when the fragments are far away from each other.

For a given value of the impact velocity, we calculate the number of fragments made up of $s$ particles. The fragment size distribution, $n(s)$, is averaged over 1000 runs by uniformly sampling the initial random angle of rotation. In fragmentation studies, it is usual to estimate the cumulative fragment size distribution divided by the fragment size $s$ [11]:

$$
N(s)=\frac{1}{s} \int_{s}^{\infty} n\left(s^{\prime}\right) d s^{\prime} .
$$

If $n(s) \sim s^{-\tau}$, then $N(s)$ exhibits the same, but less noisy, power law behaviour. 


\section{Results}

First we describe qualitatively the collision of the disc with the wall, as the impact velocity increases. At very low velocity, a slightly elastic deformation is observed at the contact with the wall, followed by the rebound of the disc without any damage. As the velocity is increased, deformation becomes irreversible and fragmentation occurs for $V>0.5$.

Three snapshots of a typical fragmentation event are shown in fig. 1. A zero initial angle is chosen in order to clearly visualize the cracks and the compression waves materialized by the potential energy of the particles. As can be seen in the top snapshot, when the disc strikes the wall, a compression wave starts to propagate from the impact zone through the disc. If the impact velocity is large enough, the compression wave produces cracks (see the middle snapshot). When the cracks coalesce and reach the surface of the disc, fragmentation takes place. The part of the initial kinetic energy which is not used to break up the system is mainly transformed into the internal and kinetic energies of the fragments. As a result of the collision, fragments fly away from each other, as illustrated by the bottom snapshot. Note that in spite of the zero initial angle and the symmetry of the lattice, irregular cracks give birth to rather rough fragments. To summarize, the crack pattern we observe is very much like those found in more sophisticated simulations 28, 29] and in two-dimensional experiments 30, 17.

It is interesting to note that the speed of sound, $c_{\text {sound }}$, in this material is much larger than the impact velocities investigated in this work $(0.5<V<4.5)$. Indeed, by observing the propagation of the compression waves (see the top snapshot in fig. 1), we roughly estimate $c_{\text {sound }} \simeq 100$. Furthermore, a calculation of the speed of sound in the harmonic approximation for a one-dimensional chain of particles interacting through the potential $\mathrm{v}(\mathrm{r})$ gives the same order of magnitude: $c_{\text {sound }}=78$.

In order to better understand the nature of this fragmentation process, we calculate the mean size of the first and second largest fragments, denoted $S_{\max 1}$ and $S_{\max 2}$ respectively. As with percolation theory, we also compute the average of the second moment of the fragment size distribution, which is related to the mean fragment size 31:

$$
m_{2}=\sum_{s} s^{2} n(s)
$$

where the sum excludes the largest fragment.

These three quantities are plotted in fig. 2 as a function of the impact velocity, at $t=100$ and at the end of the simulation at $t=500$. We clearly see that $S_{\max 1}, S_{\max 2}$ and $m_{2}$ evolve little between these two times, showing that the fragmentation process has reached a steady state. Indeed, the fragment size distribution is rapidly stabilized after the impact. Although the fragments are heated during the process, their internal energies are low and allow the evaporation of only a few particles.

As expected, $S_{\max 1}$ decreases with $V$, reflecting the increasing violence of the impact. On the other hand, both $S_{\max 2}$ and $m_{2}$ have a maximum at the same threshold velocity, $V_{t}=1.15$, as in the simulations performed by Kun, Herrmann and their collaborators [12, 28. These authors interpreted this behaviour as a sign of criticality in fragmentation. We note that the value of $V_{t}$ decreases slightly with $N$ (for instance, $V_{t} \simeq 1$ for $N=36289$ ). As is shown in fig. 2, the 

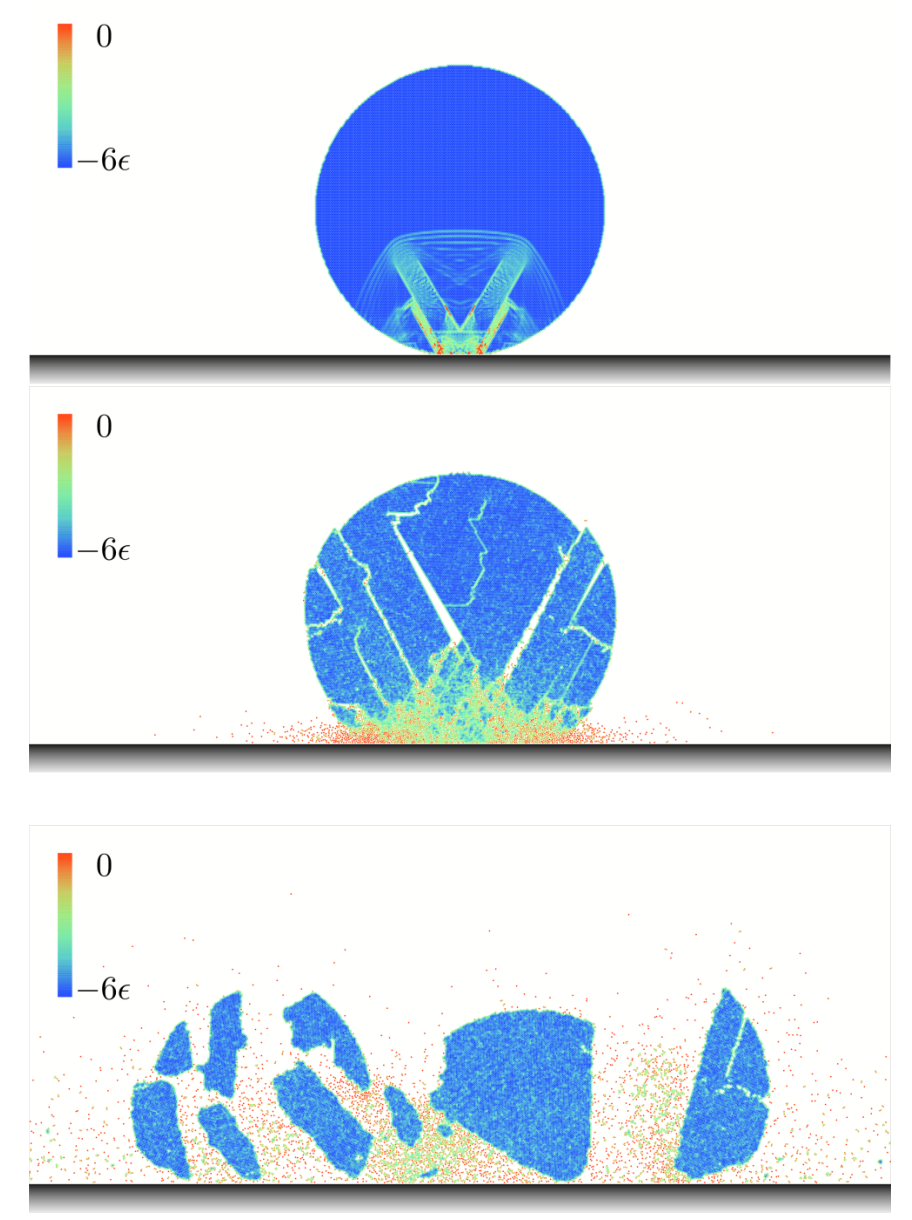

Figure 1: (colour online) Snapshots of the fragmentation of a disc of $N=36289$ particles launched with a zero initial angle and an impact velocity $V=2$, at $t=1$ (top), $t=10$ (middle) and $t=100$ (bottom). Particles are coloured according to their potential energy from $-6 \epsilon$ (blue) to 0 (red). 


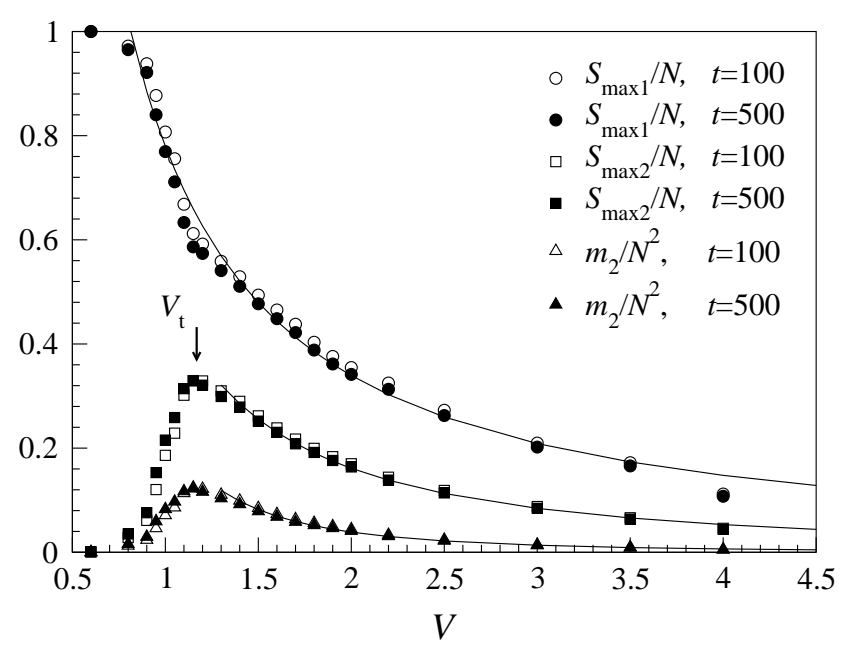

Figure 2: The first and second largest fragments and the second moment as a function of $V$ for a disc of $N=1457$ particles at time $t=100$ (open symbols) and at $t=500$ (solid symbols). The full lines correspond to power law fits (see text). The threshold velocity is indicated by an arrow.

decrease of $S_{\max 1}, S_{\max 2}$ and $m_{2}$ is well fitted by a power law $\sim V^{-\gamma}$, with an exponent $\gamma$ equal to 1.2, 1.6 and 2.6 respectively. As can be remarked, in contrast with a critical behaviour, the threshold velocity does not appear in this power law form. Moreover, the fit is very good not only in the vicinity of $V_{t}$, but also at velocities up to 3.5. It is worth noting that the power law behaviour of $S_{\max 1}$ has been reported in experimental works dealing with cubic ice $(\gamma=1.8) 32$ and aluminum spheres $(\gamma=6.6)$ 33. Moreover, by fragmenting various rock types, Matsui and his coworkers [24] showed that the exponent seems to depend mainly on the shape of the system: with $\gamma \simeq 1.4$ for spherical samples and $\gamma \simeq 3$ for cubic ones.

For $V>V_{t}$, the fragment size distribution $N(s)$ follows a power law with an exponent $\tau$ (see fig. 3). Of course, the properties and the number of small fragments $(s \leq 10)$ may depend strongly on the particular features of the system [12, 28]. However, we are mainly concerned with the large size range for which generic behaviours are expected.

To test the dependence of our results on the potential interaction, we carry out simulations with two other potentials having the same form given by eq. (1), but with a longer attraction range of $\sigma\left(v_{0}=16.29, a=13\right.$ and $\left.b=11\right)$ and $2 \sigma$ $\left(v_{0}=4, a=12\right.$ and $\left.b=6\right)$, the latter being a standard Lennard-Jones potential (see the inset of fig. 3). The fragment size distributions corresponding to these three potentials are plotted in fig. 3. Strikingly, they share the same slope, and so the same value of $\tau$, suggesting the robustness of our results. Differences occur in the small size region and for the largest fragment, the size of which increases with the range of the potential, as well as the cohesion of the disc. 


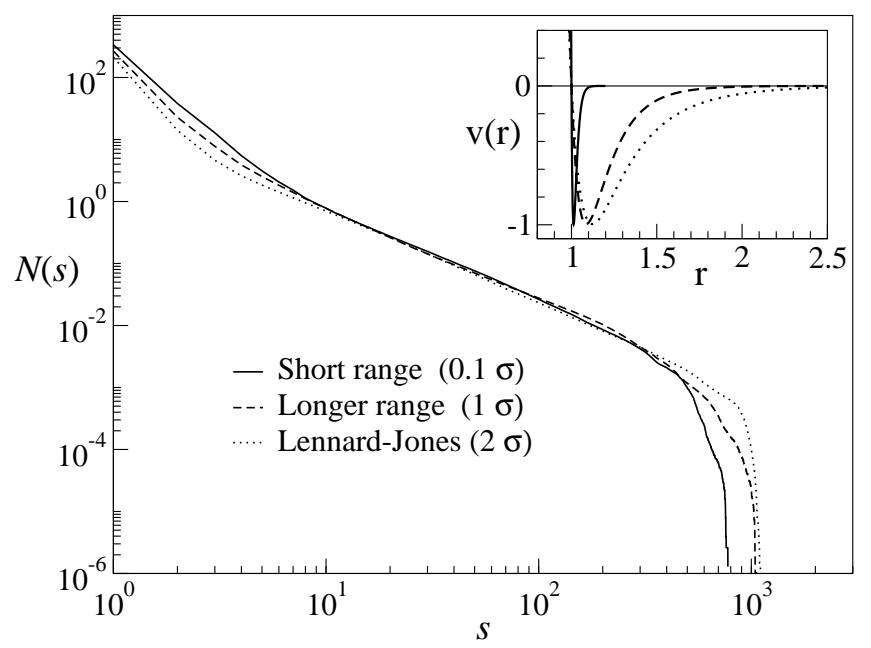

Figure 3: Fragment size distributions at $V=2$ for a disc of $N=1457$ particles and for three different interaction potentials $\mathrm{v}(\mathrm{r})$ plotted in the inset. The attraction range is given in the legend.

Furthermore, this result implies that fragmentation may be, to a certain extent, independent of the particular material as suggested by Oddershede et al. [11.

As mentioned above, the fragment size distribution exhibits a power law behaviour not only at $V_{t}$, but for all the values of the control parameter greater than $V_{t}$. What is more, we find the exponent $\tau$ to grow logarithmically with the impact velocity (see fig. 4) as

$$
\tau=\alpha \ln V+\beta,
$$

with $\alpha=0.70 \pm 0.01$ and $\beta=1.00 \pm 0.01$. Because of the decrease of $S_{\max 1}$ with increasing $V$, the power law region narrows, which enlarges the errors bars. Nevertheless, the logarithmic fit is quite sensible for $V_{t} \leq V \leq 3$.

The growth of the exponent $\tau$ with the energy imparted to the fragmenting system is clearly observed in various materials of different shapes, like glass and plaster plates [30, ceramic tubes [34, gypsum disks [18] and glass rods [10, 25]. However, as far as we know, only a few experimental papers are concerned with the exact dependence of $\tau$ on the energy. Thus, by performing impact experiments on rocks, Matsui and his collaborators 24 found the logarithmic relation given by eq. (4) with $\alpha \simeq 0.65$, regardless of the shape and rock type investigated (granite, basalt, tuff). Furthermore, Moukarzel et al. have very recently studied the fragmentation of liquid (glycerol and water) droplets by a pressurized-gas blow 23]. As can be seen in fig. 6 of ref. [23], $\tau$ has approximately a logarithmic behaviour as a function of the jet pressure, which is related to the imparted energy. The increase of $\tau$ with energy reported in other simulations of impact [26] and explosive [10] fragmentation, seems to be in agreement with eq. (4), at least at low impact energy. 


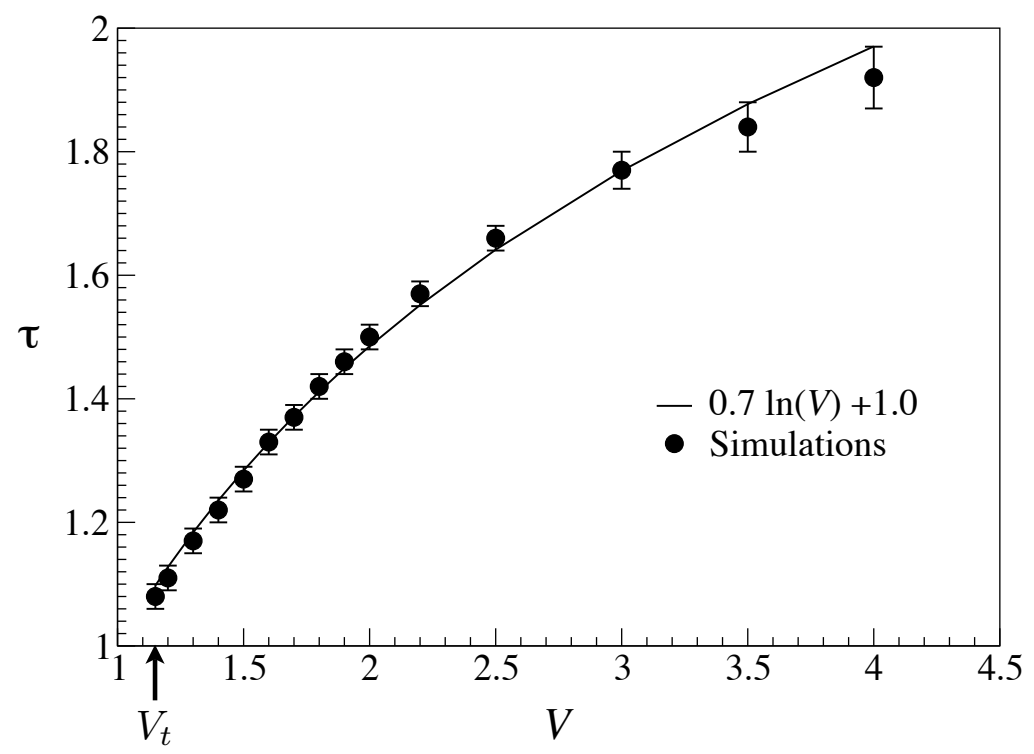

Figure 4: Exponent $\tau$ of the fragment size distribution as a function of the impact velocity for a disc of $N=1457$ particles. The full line corresponds to a logarithmic fit (see eq. (4)).

In contrast, by performing the same kinds of numerical experiments, other authors reported a virtually constant value of $\tau$ with increasing impact energy [12, 19, 20, 28, corresponding to a zero value of $\alpha$. Also, as mentioned above, simple analytical [9, 17, 18, and numerical models [27, 35, predict a value of $\tau$ which depends only on the dimension of the space.

Considering these experimental and theoretical results, we suggest that the logarithmic dependence of the exponent $\tau$ on the impact energy (or velocity) given by eq. (4) is generic of fragmentation processes. A question which naturally arises is on what parameters the coefficient $\alpha$ depends.

We now turn to the finite-size scaling properties of the fragment size distribution. It follows from the conservation of the total number of particles that:

$$
N=\sum_{s=1}^{N} s n(s) .
$$

Suppose that $n(s) \sim f(N) s^{-\tau}$, where $f$ is a function of $N$ to be determined. By taking the continuous limit (large $N$ ), we have

$$
N \sim f(N) \int_{1}^{N} s^{1-\tau} d s \sim f(N) N^{2-\tau},
$$

as long as $\tau<2$. Hence, $f(N) \sim N^{\tau-1}$, and the fragment size distribution 


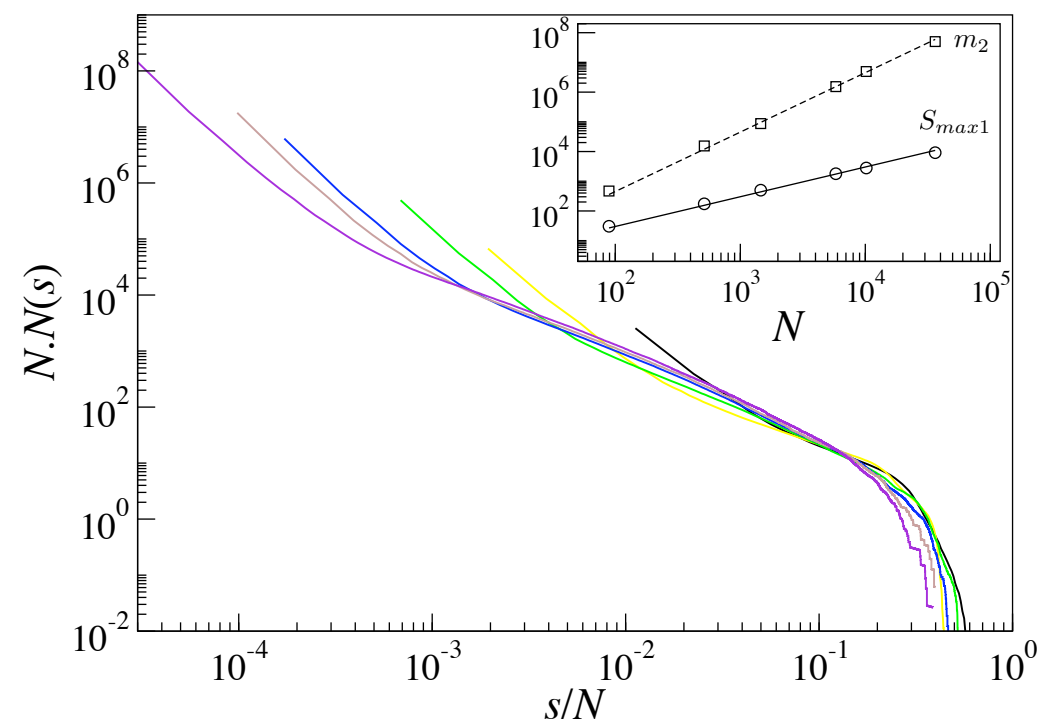

Figure 5: (colour online) Scaling collapse of the fragment size distribution at $V=2$. From right to left $N=89,515,1457,5813,10181,36289$. In the inset: $S_{\max 1}$ and $m_{2}$ are fitted by $N$ (solid lines) and $N^{2}$ (broken lines) respectively.

scales as:

$$
n(s) \sim N(s) \sim \frac{1}{N}\left(\frac{s}{N}\right)^{-\tau} .
$$

The fragment size distribution $N(s)$ defined by eq. (2) is plotted in fig. 5 for various sizes $N$ of the disc as a function of $s / N$. Considering the important surface effects at small $N$, the data collapse is good, except for the small fragment sizes, which do not follow a power law with exponent $\tau$. We find that the quality of the data collapse is better at higher impact velocities.

The validity of eq. (7) is confirmed by the scaling behaviour of the second moment of the fragment size distribution given by eq. (3). Using eq. (7), we obtain (with $\tau<3$ ):

$$
m_{2} \sim N^{\tau-1} \int_{1}^{N} s^{2-\tau} d s \sim N^{2} .
$$

As illustrated in the inset of fig. 5 , the scaling behaviour predicted by eq. (8) is in very good agreement with the simulation data. Furthermore, the size of the largest fragment scales nicely with the total number of particles, that is $S_{\max 1} \sim N$. It must be emphasized that the scaling behaviour of $S_{\max 1}$ and $m_{2}$ is independent of $\tau$, and is then observed for all the velocities larger than $V_{t}$ investigated in this work. 


\section{Discussion}

To summarize, this simple model provides a general framework for investigating the various parameters that may play a role in fragmentation processes. As we have seen, our results do not depend on the range of attraction of the interaction potential, which suggests a certain universality in fragmentation of solid bodies. Qualitatively, the propagation of cracks and the crack pattern obtained in our simulations are akin to the ones observed experimentally in platelike objects.

The power law behaviour of the fragment size distribution and the maximum of its second moment seem to provide evidence that fragmentation is a continuous phase transition, similar to a percolation transition, as suggested by Kun and Herrmann [12. However, in contrast to continuous phase transitions, the power law is observed, not only at the threshold velocity, but also at all velocities above $V_{t}$. Moreover, the scaling behaviour of the fragment size distribution is simply inferred from the conservation of the number of particles and does not reveal a critical behaviour. We note that these properties are very similar to the avalanche phenomena associated with hysteresis loops, in which Sethna and his collaborators [37] found scaling and power law behaviours in a large region near the critical point. However, this analogy has to be confirmed by thorough analysis.

Furthermore, we find that the exponent of the fragment size distribution increases as a logarithmic function of the imparted energy, in agreement with experiments on rocks and on liquid droplets. This result casts some doubts upon the existence of universality classes in fragmentation, in the strict sense of continuous phase transitions, but it sheds light on the scattering of the values of $\tau$ measured experimentally. Besides, we expect this logarithmic relation to be generic in fragmentation phenomena.

We thank H.J. Herrmann for helpful conversations, P. Viot and H. Hietala for critically reading the manuscript.

\section{References}

[1] Beysens D., Campi X.and Pefferkorn E., Proceedings of the workshop Fragmentation Phenomena, World scientific, Singapore (1995).

[2] Matsui T. and Mizutani H., Nature 270, 506 (1977).

[3] Turcotte D.L., J. Geophys. Res.91, 1921 (1986).

[4] Kaminski E. and Jaupart C., J. Geophys. Res.103, 29759 (1998).

[5] Elliott J.B. et al., Phys. Rev. C 67, 024609 (2003).

[6] Campi X., Krivine H., Sator N. and Plagnol E., Eur. Phys. J. D 11, 233 (2000).

[7] Frances C. and Laguerie C., Powder Technol. 99, 147 (1998). 
[8] Oddershede L., Meibom A. and Bohr J., Europhys. Lett. 43, 598 (1998).

[9] Meibom A. and Balslev I., Phys. Rev. Lett. 76, 2492 (1996).

[10] Ching E.S.C., Yiu Y.Y. and Lo K.F., Physica A 265, 119 (1999).

[11] Oddershede L., Dimon P. and Bohr J., Phys. Rev. Lett. 71, 3107 (1993).

[12] Kun F. and Herrmann H.J., Phys. Rev E 59, 2623 (1999).

[13] Åström J.A., Holian B.L. and Timonen J., Phys. Rev. Lett. 84, 3061 (2000).

[14] Sator N., Phys. Rep. 376, 1 (2003).

[15] Farizon B. et al., Phys. Rev. Lett. 81, 4108 (1998).

[16] Boyer D., Tarjus G. and Viot P., Phys. Rev E 51, 1043 (1995).

[17] Kadono T. and Arakawa M., Phys. Rev. E 65, 035107(R) (2002).

[18] Åström J.A., Linna R.P., Timonen J., Møller P.F. and Oddershede L., Phys. Rev. E 70, 026104 (2004).

[19] Diehl A.,Carmona H.A., Araripe L.E., Andrade J.S. Jr. and Farias G.A. Phys. Rev E 62, 4742 (2000).

[20] Araripe L.E., Andrade J.S. Jr. and Costa Filho R.N., Phys. Rev E 71, 036119 (2005).

[21] Frenkel D. and Smit B., Understanding molecular simulation: From algorithms to Applications, Academic Press, Oxford (2001).

[22] Hill T.L., J. Chem. Phys. 23, 617 (1955).

[23] Moukarzel C.F., Fernández-Sabido S.F. and Ruiz-Suárez J.C., Phys. Rev. E 75, 061127 (2007).

[24] Matsui T., Waza T., Kani K. and Suzuki S., J. Geophys. Res. 87, 10968 (1982).

[25] Ishii T. and Matsushita M., Jour. Phys. Soc. Jpn. 61, 3474 (1992).

[26] Myagkov N.N. and Shumikhin T.A., Physica A 358, 423 (2005).

[27] Inaoka H., Toyosawa E. and Takayasu H., Phys. Rev. Lett. 78, 3455 (1997).

[28] Behera B., Kun F., McNamara S. and Herrmann H.J., J. Phys.: Condens. Matter 17, S2439 (2005).

[29] Thornton C., Yin K.K. and Adams M.J., J. Phys. D: Appl. Phys. 29, 424 (1996).

[30] Kadono T., Phys. Rev. Lett. 78, 1444 (1997).

[31] Stauffer D. and Aharony A., Introduction to percolation theory, Taylor and Francis, London/Bristol (1994).

[32] Arakawa M., Icarus 142, 34 (1999). 
[33] Piekutowski A.J., Int. J. Impact Engng 17, 627 (1995).

[34] Katsuragi H., Ihara S. and Honjo H., Phys. Rev. Lett. 95, 095503 (2005).

[35] Åström J.A., Ouchterlony F., Linna R.P. and Timonen J., Phys. Rev. Lett. 92, 245506 (2004).

[36] Wittel F., Kun F., Herrmann H.J. and Kröplin B.H., Phys. Rev. Lett. 93, 035504 (2004).

[37] Perković O., Dahmen K.A. and Sethna J.P., Phys. Rev. B 59, 6106 (1999). 